\title{
Shock and blast waves mitigation
}

\author{
A. Hadjadj • O. Sadot
}

Published online: 3 January 2013

(C) Springer-Verlag Berlin Heidelberg 2012

\section{Introduction}

Shock and blast waves propagation in complex media is one of the most important topics of current shock wave research. In the past, most of the shock wave researches dealt with shock phenomena in relatively simple configurations and fluid flow conditions. However, accounting for modern developments of shock research and its evolution into interdisciplinary applications, there is a need to study wave phenomena in complex environments such as those consisting of complex constituents. Furthermore, the prediction of damage caused by blast waves, generated by largescale explosions (see Figs. 1 and 2), either from terrorist attacks or industrial hazards has been a research problem that needs further understanding from disaster prevention point of view. These important issues have two common features: (1) understand the complex flow field induced by blast and shock waves that pass through complex media and (2) help designing new devices for protection against blast loading.

While preparing this thematic issue, we have been deeply saddened by the death of our colleague and friend Dr. Alexander (Alex) Britan, from Ben-Gurion University of the Negev (Israel), who passed away couple of months before the publication of the current issue. Given the degree of his implication in this work, and the importance of his contribution to the field of blast mitigation and filtration, we wish to dedicate this issue to his memory.

\section{A. Hadjadj (凶)}

National Institute of Applied Sciences INSA of Rouen CORIA

UMR 6614 CNRS, 8 Avenue de l'Université Saint Etienne du

Rouvray, Rouen 76800, France

e-mail: hadjadj@coria.fr

O. Sadot

Shock Tube Laboratory, Mechanical Engineering Department, Ben-Gurion University of the Negev, Beer-Sheva, Israel

e-mail: sorens@bgu.ac.il

Usually, experimental investigations on the response of structures to shock and blast waves are performed in fullscale field tests. Although these experiments are limited in their number due to their high-cost and low-repeatability, they are very useful as they provide real information on the peak pressure and impulse values and the associated nonlinear effects that steepen the waveform, decreasing thereby the shock rise time. The analysis of large-scale explosively generated shock waves may also aid the engineer in quickly assessing air-blasts propagation and their interaction with the surrounding environment (air, dust, granular, liquid or porous media).

Interms of blast mitigation, different type of protective layers can absorb energy either by plastic deformation or by buckling. For instance, the response of metallic foams to high stress rate has received increased interest due to their highenergy absorption capabilities during their deformation. It is believed that the high energy absorbing capabilities that characterize these materials will enable engineers to use metallic foams as protective layers against explosion-induced blast waves. Aluminium foams are an effective example of those materials. Different types of absorbing-energy materials exist as well, e.g. aqueous foams, where different experiments are conducted to investigate the mechanical and thermodynamic processes involved in the interaction. In the case of aqueous foams (see Fig. 3), existing information on the foam collapse as well as heating and evaporating the liquid phase behind the blast wave is rather restricted. To analyze the strong heat transfer at the blast/foam interface within the fireball and/or behind the expansion fan containing the blast products, more comprehensive studies are required. Also, the role of the fluctuations of the thermodynamic quantities in the post shock wave flow in foam is a challenging problem from the theoretical point of view. Since the pressure dilatation correlation is very sensitive to the increased level of pressure fluctuations, 


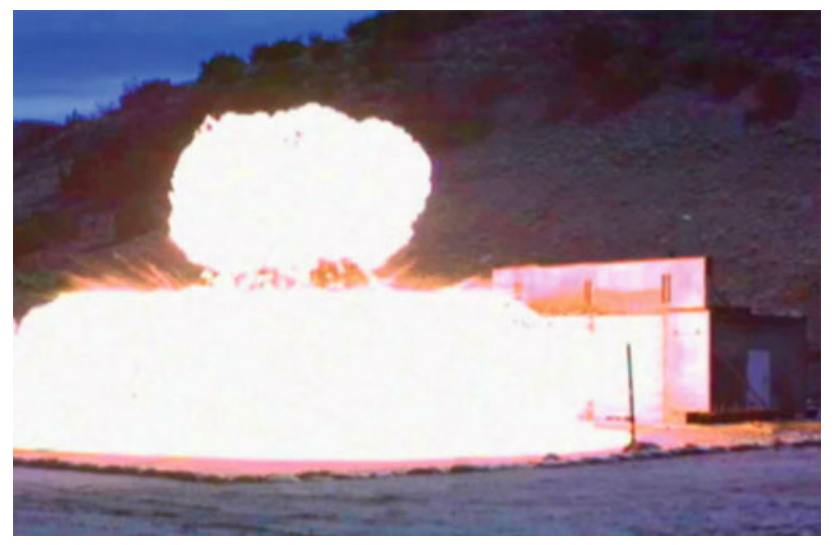

Fig. 1 Large-scale explosion

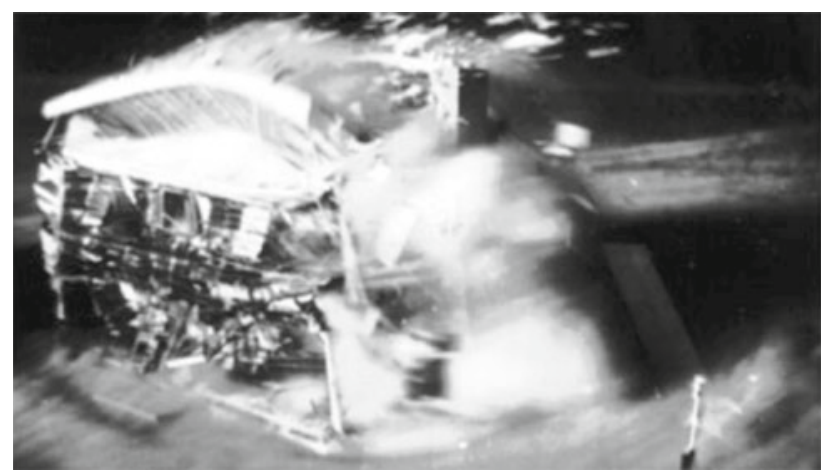

Fig. 2 Structure damage caused by blast threats

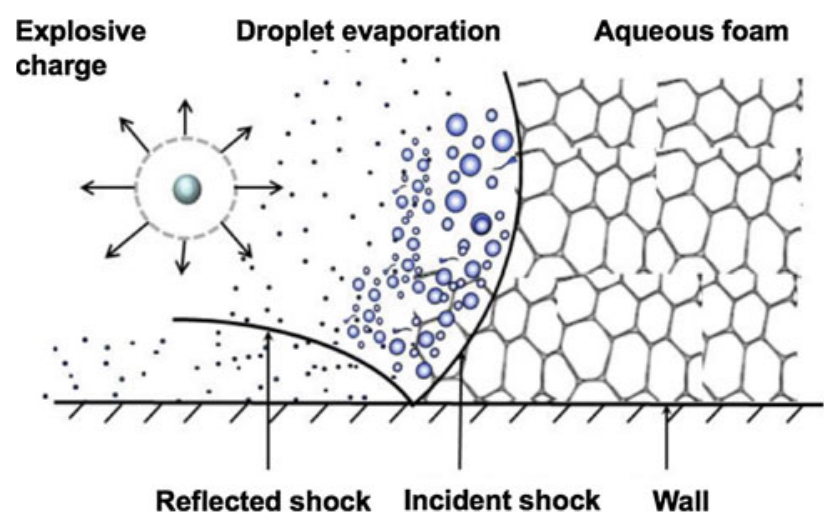

Fig. 3 Interaction of blast wave with aqueous foam. Courtesy of A. Chinnayya, University of Rouen, France

the associated compressibility effects are very complex to analyze.

For more fundamental studies, small-scale experiments have been conducted in laboratory facilities such as shock tubes or equivalent systems. The flexibility of the laboratory experiments can be explored to better understand the governing physical processes by testing experimental setups with gradually increased complex geometries. For this purpose,
Shock

wave

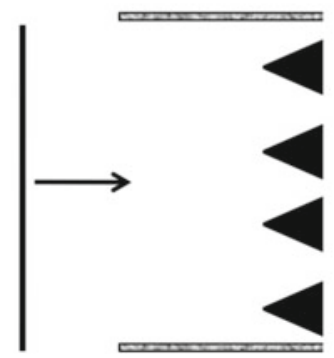

Fig. 4 Shock wave interaction with solid obstacles

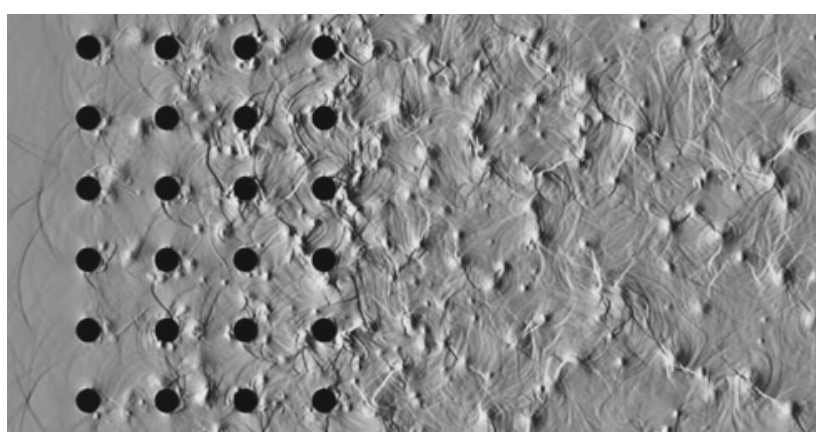

Fig. 5 Numerical schlieren picture showing the post-shock flow oscillations due to vortex shedding and baroclinic instabilities downstream of the obstacles. Courtesy of Chaudhuri et al. [39]

the rate of shock attenuation has been intensively studied numerically and experimentally using different geometrical means, such as perforated screens, grids, orifice plates, or rigid obstacles. An example of a shock wave interacting with triangular and cylindrical matrices of obstacles is shown in Figs. 4 and 5.

In spite of many studies on the subject [1-30], the mechanisms of shock propagation in such complex media still need further research.

\section{Thematic issue on SBWM}

The thematic issue on shock and blast waves mitigation (SBWM) focuses on the integration of physical analysis, modeling, and experiments for the study of blast waves interactions with inhomogeneous flows, and helps to provide a basis for future work in this area. This issue includes seven original or review papers from specialists in the various aspects of SBWM (physical description, advanced measurements, and numerical simulations). Roughly half of the contributions are experimental investigations, and the other half is dedicated to numerical simulations. It is should be mentioned that all manuscripts have been strictly peer-reviewed according to the Shock Waves Journal procedure and policy (with at least two- or three- anonymous reviewers for each paper). 
In the framework of these studies, several experimental techniques have been used with different flow configurations (polymer, dry or aqueous foams) to elucidate the phenomenon of shock attenuation. For example, Petel et al. [31] undertook a series of experimental investigations of blast/shock wave mitigation through polymer (open and closed-cell) foams. The focus of the paper is the precursor wave during dynamic compaction of cellular materials. The investigation highlights the different approaches that have been adopted in previous investigations, and increases the understanding of the mechanics/physics of dynamic compaction of foams. This includes the difference between foams and monolithic elastic-plastic materials in terms of wave propagation and attenuation. In Britan et al. [32], an experimental study of shock wave impact on an aqueous foam column was conducted in shock tube tests. Useful results were obtained in identifying the effect of wave acceleration and on the effect of liquid fraction as well as on the wave thickness. Interesting conclusions were drawn on the influence of the contact between the foam layer adjacent to the wall and the sensing element of the transducer. In addition, Britan et al. [33] presented a review paper in which they summarized the essential features of aqueous-foam barriers against blast waves impact encountered in both shock-tube experiments and full-scale field tests. The modeling aspect of blast/shock waves mitigation was also discussed. The paper presented by Del-Prete et al. [34] describes experimental and numerical investigations on the mitigation of blast waves using dry aqueous foams. Different tests were conducted in sub- and full-scale configurations using three different masses of explosive. To support their experiments, a multiphase approach was used to model the aqueous foam and its interaction with a blast wave issued from the explosion. Comparison of the results shows a good correlation between the experimental and computational results.

From a computational and modeling point of view, the paper presented by ElBaz et al. [35] is concerned with a computational study of shock propagation in heterogeneous media. The media were considered as a wetted foam and modeled as a regular staggered row of lattice parallel fibers immersed in a fluid having a strong density contrast. It was found that the shock speed is enhanced in comparison to its equivalent speed in a homogeneous medium. The study also showed the importance of accounting for the turbulent kinetic energy when modeling the post-shock instabilities. Al-Qananwah et al. [36] reported numerical results of shock propagation and interaction with structured porous material at the nano-scale level. The computations use molecular dynamics (MD) simulations. Similar approach has been used previously by Sen and Sinkovits [37,38] for the shock wave and sound wave mitigation in granular columns.

Finally, Chaudhuri et al. [39] used an advanced NavierStokes code with a large-eddy simulation technique based on an immersed boundary method to discuss the somewhat geometrical effect of different solid obstacles and matrices arrangement in shock attenuation. In addition, it was shown that the two-dimensional flow induced by the shock wave is rapidly converted into three-dimensional turbulent flow. Following this scenario, the energy can be transferred from direct kinetic mode to rotational (baroclinic) energy and then dissipated into molecular effects.

Acknowledgments We would like to thank all authors for their valuable contributions, and also reviewers for their useful comments and assistance. Special thank to Prof. Evgeny Timofeev who helped us with the technical management of this issue.

\section{References}

1. Baer, M.R.: A numerical study of shock wave reflections on low density foam. Shock Waves 2, 121-124 (1992)

2. Britan, A., Ben-Dor, G., Igra, O., Shapiro, H.: Shock waves attenuation by granular filters. Int. J. Multiphase Flow 27, 617-634 (2001)

3. Britan, A., Kivity, Y., Ben-Dor, G.: Passive deflection for attenuation of shock waves. In: Proceedings of 26th International Symposium Shock Waves, Bangalore, India (2005).

4. Britan, A., Kivity, Y., Ben-Dor, G.: Blast wave discharge into a shelter with inlet cheveron. In: Proceedings of 26th International Symposium Shock Waves, Gottingen, Germany. Springer, Berlin (2007)

5. Cooper, W.P.: Explosive Engineering. Wiley, New York (1996)

6. Kinney, G.F., Graham, K.J.: Explosive Shocks in Air, 2nd edn. Springer, Berlin (1985)

7. Zukas, J.A., Walters, P.W.: Explosive Effects and Applications. Springer, Berlin (2003)

8. Gibson, L.J., Ashby, M.F.: Cellular Solids: Structure and Properties. Pergamon Press, Oxford (1988)

9. Kitagawa, K., Jyonouchi, T., Yasuhara, M.: Drag difference between steady and shocked gas flows passing through a porous body. Shock Waves 11, 133-139 (2001)

10. Kitagawa, K., Yokoyama, M., Yasuhara, M.: Attenuation of shock wave by porous materials. In: Proceedings of 24th International Symposium on Shock Waves, China, vol. 2, pp. 1247-1252 (2004)

11. Kitagawa, K., Takayama, K., Yasuhara, M.: Attenuation of shock waves propagation in polyurethane foams. Shock Waves 15(6), 437-445 (2006)

12. Levy, A., Ben-Dor, G., Sorek, S.: Numerical investigation of the propagation of shock waves in rigid porous materials: flow field behavior and parametric study. Shock Waves 8, 127-137 (1998)

13. Olim, M., van Dongen, M.E.H., Kitamura, T., Takayama, K.: Numerical simulation of the propagation of shock waves in compressible open-cell porous foams. Int. J. Multiphase Flow 20-3, 557-568 (1994)

14. Skews, B.W., Atkins, M.D., Seitz, M.W.: The impact of a shock wave on porous compressible foams. J. Fluid. Mech. 253, 245-265 (1993)

15. Seitz, M., Skews, B.: Effect of compressible foam properties on pressure amplification during shock wave impact. Shock Waves 15(34), 177-197 (2006)

16. Skews, B.W.: Shock wave interaction with porous plates. Exp. Fluids 39, 875-884 (2005)

17. Inoue, O., Imuta, S., Multon, B.E., Takayama, K.: Computational study of shock wave focusing in a log-spiral duct. Shock Waves 5(3), 183-188 (2005) 
18. Rogg, B., Hermann, D., Adomeit, G.: Shock-induced flow in regular arrays of cylinders and packet beds. Int. J. Heat Mass Transfer 28(12), 2285-2298 (1985)

19. Sasoh, A., Matsuoka, K., Nakashio, K., Timofeev, E., Takayama, K., Voinovich, P., Saito, T., Hirano, S., Ono, S., Makino, Y.: Attenuation of weak shock along pseudo-perforated walls. Shock Waves 8(3), 149-159 (1998)

20. Reichenbach, H., Kuhl, A.L.: Shock-induced turbulent flow in baffle system. In: Proceeding 19th International Symposium Shock Waves, Marseille, France. Springer, Berlin (1993).

21. Bakken, J., Slungaard, T., Engebretsen, T., Cristensen, S.O.: Attenuation of shock waves by granular filters. Shock Waves 13(1), 33-40 (2003)

22. Kleine, H., Dewey, J.M., Ohashi, K., Mizukaki, T., Takayama, K.: Studies of the TNT equivalence of silver azide charges. Shock Waves 13(2), 123-138 (2003)

23. Dewey, J.M.: Air velocity in blast waves from TNT explosions. Proc. Roy. Soc. A 279(1378), 366-385 (1964)

24. Dewey, J.M.: Expanding spherical shocks (blast waves). In: BenDor, G., Igra, O., Elperin, E. (eds.) Handbook of Shock Waves 2, pp. 441-481. Academic Press, London (2001)

25. Gelfand, B.E.: Attenuation of blast waves in two-phase mixtures. In: Proceedings of the International Symposium on Interdisciplinary Shock Wave Research, ISISW, 22-24 March, pp. 150166. Sendai, Japan (2004). http://iswi.nuae.nagoya-u.ac.jp/ISISW/ ISISW.html

26. Needham, C.E.: Blast Waves. Springer, Berlin (2006)

27. Britan, A.B., Vasilev, E.I., Kulikovskii, V.A.: Modeling the process of shock-wave attenuation by a foam screen. Combust. Explos. Shock Waves 30(3), 389-396 (1994)

28. Britan, A., Liverts, M., Ben-Dor, G.: Shock wave propagation through wet particulate foam. Colloids Surf. A Physicochem. Eng. Aspects 382(1-3), 145-153 (2011)
29. Britan, A., Zinovik, I., Levin, V.: Breaking up foam with shock waves. Combust. Explos. Shock Waves 28(5), 550-557 (1992)

30. Britan, A.B., Ben-Dor, G., Shapiro, H., Liverts, M., Shreiber, I.: Drainage effects on shock wave propagating through aqueous foams. Colloids Surf. A Physicochem. Eng. Aspects 309(1-3), 137-150 (2007)

31. Petel, 0, Ouellet, S., Higgins, A., Frost, D.: The elastic-plastic behaviour of foam under shock loading. Shock Waves (2013). doi:10.1007/s00193-012-0414-7

32. Britan, A., Shapiro, H., Liverts, M., Ben-Dor, G.: Macromechanical modeling of blast-wave mitigation in foams. Part II: reliability of pressure measurements. Shock Waves (2013). doi:10. 1007/s00193-012-0402-y

33. Britan, A., Shapiro, H., Liverts, M., Ben-Dor, G., Chinnayya, A., Hadjadj, A.: Macro-mechanical modeling of blast-wave mitigation in foams. Part I: review of available experiments and models. Shock Waves (2013). doi:10.1007/s00193-012-0417-4

34. Del Prete, E., Chinnayya, A., Domergue, L., Hadjadj, A., Hass, J.F.: Blast wave mitigation by dry aqueous foams. Shock Waves (2013). doi:10.1007/s00193-012-0400-0

35. ElBaz, D., Canaud, B., Ballereau, P., Dias, F.: Shock propagation in regular wetted arrays of fibers. Shock Waves (2013) doi:10.1007/ s00193-012-0424-5

36. Al-Qananwah, A.K., Koplik, J., Andreopoulos, Y.: Shock wave interactions with nano-structured materials: a molecular dynamics approach. Shock Waves (2013). doi:10.1007/s00193-012-0397-4

37. Sinkovits, R.S., Sen, S.: Nonlinear dynamics in granular columns. Phys. Rev. Lett. 74, 2686-2689 (1995)

38. Sen, S., Sinkovits, R.S.: Sound propagation in impure granular columns. Phys. Rev. E 54, 6857-6865 (1996)

39. Chaudhuri, A., Hadjadj, A., Sadot, O., Ben-Dor, G.: Numerical study of shock-wave mitigation through matrices of solid obstacles. Shock Waves (2013). doi:10.1007/s00193-012-0362-2 